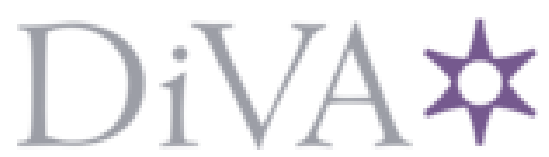

http://www.diva-portal.org

This is the published version of a paper presented at The 16th International Conference on Climbing and Walking Robots and the Support Technologies for Mobile Machines (CLAWAR 2013), Sydney, Australia, 14-17 July, 2013.

Citation for the original published paper:

Khandelwal, S., Chevallereau, C. (2013)

Estimation of the Trunk Attitude of a Humanoid by Data Fusion of Inertial Sensors and Joint Encoders.

In: Kenneth J. Waldron, Mohammad O. Tokhi \& Gurvinder S. Virk (ed.), Nature-Inspired Mobile Robotics (pp. 822-830). Singapore: World Scientific

N.B. When citing this work, cite the original published paper.

Permanent link to this version:

http://urn.kb.se/resolve?urn=urn:nbn:se:hh:diva-23363 


\title{
Estimation of the Trunk Attitude of a Humanoid by Data Fusion of Inertial Sensors and Joint Encoders
}

\author{
SIDDHARTHA KHANDELWAL \\ Intelligent Systems Laboratory, Halmstad University, \\ Halmstad, Box 823, S-301 18, Sweden \\ E-mail: siddhartha.khandelwal@hh.se \\ CHRISTINE CHEVALLEREAU \\ IRCCYN, CNRS, Ecole Centrale de Nantes, \\ Nantes, Cedex 3 - 44321, France \\ E-mail: christine.chevallereau@irccyn.ec-nantes.fr
}

\begin{abstract}
The major problem associated with the walking of humanoid robots is to maintain its dynamic equilibrium while walking. To achieve this one must detect gait instability during walking to apply proper fall avoidance schemes and bring back the robot into stable equilibrium. A good approach to detect gait instability is to study the evolution of the attitude of the humanoid's trunk. Most attitude estimation techniques involve using the information from inertial sensors positioned at the trunk. However, inertial sensors like accelerometer and gyro are highly prone to noise which lead to poor attitude estimates that can cause false fall detections and falsely trigger fall avoidance schemes. In this paper we present a novel way to access the information from joint encoders present in the legs and fuse it with the information from inertial sensors to provide a highly improved attitude estimate during humanoid walk. Also if the joint encoders' attitude measure is compared separately with the IMU's attitude estimate, then it is observed that they are different when there is a change of contact between the stance leg and the ground. This may be used to detect a loss of contact and can be verified by the information from force sensors present at the feet of the robot. The propositions are validated by experiments performed on humanoid robot NAO.
\end{abstract}

Keywords: Humanoid walking; Dynamic equilibrium; Attitude estimation; Instability detetection; Sensor fusion; Inertial sensors; Joint encoders.

\section{Introduction}

There has been increasing academic and commercial interest in humanoid robots for various applications since the recent significant advancements in robot control technology. An effective trunk attitude control is one of the 
most important factors for a humanoid to continue dynamically stable walking which is necessary to accomplish any given task. Most indoor robots use inertial sensors and vision, or other external signals and cues, for determining their position and orientation. In most cases, humanoid robots like NAO use MEMS based inertial sensors to calculate its attitude due to cost, payload and other limitations. Most inertial measurement units (IMUs) consist of 3-axis gyros and accelerometers which measure the angular rate and specific forces of the vehicle respectively. Hence these IMUs do not need external sources for attitude measurement. Conventionally, attitude is computed by integrating the angular rate obtained from gyro readings. However, this method is inappropriate as the gyro bias error makes the attitude error to diverge. ${ }^{1}$ On the other hand the attitude calculated by accelerometers is heavily influenced by mechanical disturbances caused by the movement of the robot. Accordingly, a number of fusion algorithms have been developed like Complementary filter method, ${ }^{2}$ direction cosine matrix method, ${ }^{3}$ Euler angle update method ${ }^{4}$ and modified versions of linear and extended Kalman Filter (EKF $)^{567}$ in particular. In all these methods, different filtering or fusion techniques are applied to minimize the error in IMU measurements. However, accuracy of the attitude thus calculated is still poor considering the high level of noise present in the inertial sensor readings. There is a need to gather more information from other sensors to improve the accuracy of the estimated attitude for a better trunk control capability. We propose that the information from joint encoders present in the legs can be used to measure the trunk's attitude during different phases of humanoid walk. This extra sensor measurement, which is readily avaliable, is combined with the IMU data using an EKF to provide a highly improved estimate of the trunk's attitude. Another outcome is that if the joint encoders' attitude measure is compared separately with the IMU's attitude estimate, then it is observed that they are different when there is a change of contact between the stance leg and the ground. This may be used to detect a loss of contact and can be compared and validated by the information from force sensors located at the feet of the humanoid.

In this paper, the process model of the EKF is developed using the gyro data. Then, indivudial measurement models are developed for accelerometer and joint encoders for different phases of the humanoid walk. These individual measurement models are then combined to construct a general Measurement Model for the EKF. Different experiments are done on humanoid robot NAO and the results are discussed to show the relevance of using joint encoder data for obtaining an improved trunk attitude estimate. 


\section{EKF for Estimating the Trunk Attitude}

A Kalman filter provides a simple yet effective way to fuse all sensor information that is provided to it. ${ }^{8}$ We use an EKF as the described process is non-linear. The notations of Welch and Bishop ${ }^{5}$ are used for constructing the EKF.

The orientation of the non-inertial frame $n$, attached to the trunk of the humanoid, relative to the inertial reference frame $b$, attached to the ground, can be described in terms of three consecutive rotations through three body-referenced Euler angles. ${ }^{9}$ These are commonly written as $\psi, \theta$ and $\phi$ which are the yaw, pitch and roll angles respectively. In this paper, the states variables of the EKF provide a measure of only $\theta$ and $\phi$. They are enough to detect the instability at the trunk. The yaw angle diverges as there is no other measurement to compensate for the gyro bias erros. ${ }^{4}$ The overall transformation ${ }^{n} R_{b}$ from frame $b$ to $n$ is calculated ${ }^{49}$ and the elements of last row of ${ }^{b} R_{n}$ are chosen as the state variables $\left(x_{1}, x_{2}, x_{3}\right)$ :

$$
\begin{aligned}
& { }^{n} R_{b}={ }^{b} R_{n}^{T}=\left[\begin{array}{ccc}
1 & 0 & 0 \\
0 & \cos \phi & \sin \phi \\
0 & -\sin \phi & \cos \phi
\end{array}\right]\left[\begin{array}{ccc}
\cos \theta & 0 & -\sin \theta \\
0 & 1 & 0 \\
\sin \theta & 0 & \cos \theta
\end{array}\right]\left[\begin{array}{ccc}
\cos \psi & \sin \psi & 0 \\
-\sin \psi & \cos \psi & 0 \\
0 & 0 & 1
\end{array}\right] \\
& \therefore \quad x_{1}=-\sin \theta ; \quad x_{2}=\sin \phi \cos \theta ; \quad x_{3}=\cos \phi \cos \theta
\end{aligned}
$$

Angles $\theta$ and $\phi$ are calculated from the state variables as $\theta=-\sin ^{-1} x_{1}$ and $\phi=\tan ^{-1} x_{2} / x_{3}$ respectively.

\subsection{Process Model of the $\mathrm{EKF}$}

The process model is based on gyro measurements. There exists a relationship between the angular rates in the $x, y, z$ axis, i.e $\omega_{x}, \omega_{y}, \omega_{z}$ and the time rate-of-change of the Euler angles: ${ }^{9}$

$$
\left[\begin{array}{c}
\dot{\phi} \\
\dot{\theta} \\
\dot{\psi}
\end{array}\right]=\left[\begin{array}{ccc}
1 & \sin \phi \tan \theta & \cos \phi \tan \theta \\
0 & \cos \phi & -\sin \phi \\
0 & \sin \phi \sec \theta & \cos \phi \sec \theta
\end{array}\right]\left[\begin{array}{l}
\omega_{x} \\
\omega_{y} \\
\omega_{z}
\end{array}\right]
$$

Differentiating the state variables in eq. (2) and using eq. (3), the state derivatives can be written in matrix form (refer eq. (4)) where $u$ is skewsymmetric matrix of the angular rates and $w$ represents process noise. This can then be expressed in discrete time as shown in eq. (5)(for $i=1,2,3)$.

$$
\begin{aligned}
{\left[\begin{array}{lll}
\dot{x_{1}} & \dot{x_{2}} & \dot{x_{3}}
\end{array}\right]^{T} } & =\left[u_{\text {skew-symmetric }(\omega)}\right]\left[\begin{array}{lll}
x_{1} & x_{2} & x_{3}
\end{array}\right]^{T}+w(t) \\
\Rightarrow x_{i, k} & =\left[I+\left(u_{k-1}+w_{k-1}\right) \Delta t\right] x_{i, k-1} \\
& =f_{i}\left(x_{i, k-1}, u_{k-1}, w_{k-1}\right)
\end{aligned}
$$


The state variables so chosen meet a constraint, i.e. $x_{1}^{2}+x_{2}^{2}+x_{3}^{2}=1$. Imposing this constraint we obtain the process model as:

$$
x_{i, k}=\frac{f_{i}\left(x_{i, k-1}, u_{k-1}, w_{k-1}\right)}{\left\|f_{i}\left(x_{i, k-1}, u_{k-1}, w_{k-1}\right)\right\|}
$$

\subsection{Measurement Model of the $\mathrm{EKF}$}

Individual measurement models are constructed for accelerometer and joint encoders for single and double support and then combined based on the type of support the humanoid has with the ground to form a general measurement model. (All variables of the measurement model are expressed in discrete time and have a subscript $k$.)

\subsubsection{Accelerometer}

The accelerometer is not used to measure the real acceleration of the robot but to measure the trunk attitude assuming that the IMU (or trunk) is in steady state. Here it measures only the gravity vector expressed in the frame $n$, as this frame is attached to the IMU. The effect of trunk velocity and acceleration are included in accelerometer noise $v_{\text {acc }}$. Thus the accelerometer measurements can be expressed as a function of the state variables (using eq. (2)) which gives the accelerometer measurement model as:

$$
\begin{aligned}
{\left[a c c_{x} a c c_{y} a c c_{z}\right]^{T} } & =-g[-\sin \theta \sin \phi \cos \theta \cos \phi \cos \theta]^{T}+v_{\mathrm{acc}} \\
& =-g\left[x_{1} x_{2} x_{3}\right]^{T}+v_{\mathrm{acc}} \\
\Rightarrow g_{\mathrm{Acc}}\left(a c c_{x}, a c c_{y}, a c c_{z}\right) & =h_{\mathrm{Acc}}\left(x_{1}, x_{2}, x_{3}, v_{\mathrm{acc}}\right)
\end{aligned}
$$

\subsubsection{Joint Encoders - Single Support (SS)}

The joint values are provided by the joint encoders present at the hip and legs. Modelling of the humanoid is done using the DH method ${ }^{10}$ and the notation of Khalil ${ }^{11}$ is used. (For eg. refer Fig. 1a.) In SS, the humanoid is considered as a serial structure from the stance foot to the trunk. By computing the DH table for each leg, the transformation matrix ${ }^{b} T_{n}$ is calculated using the Direct Geometric Model (DGM). It must be noted that the rotation matrices ${ }^{b} R_{0}$ and ${ }^{l f} R_{n}$ must be known (frame $l f$ is attached to the last link of the leg). The rotation matrix ${ }^{b} R_{n}$ is simply the first 3 rows and columns of ${ }^{b} T_{n}$ :

$$
{ }^{b} R_{n, \text { Leg }}={ }^{b} R_{0}{ }^{0} R_{l f}\left(q_{i}, v_{\text {joint }}\right){ }^{l f} R_{n}
$$


where $q_{i}$ are the joint angles used to calculate the DGM and $v_{\text {joint }}$ is the noise at each joint. This rotation can also be represented using Euler angles. Hence comparing the last row of ${ }^{b} R_{n, \text { Leg }}\left(R_{31}, R_{32}, R_{33}\right)$ with last row of ${ }^{b} R_{n}$ in eq. (1) and substituting state variables using eq. (2); the joint encoder model for SS is obtained as:

$$
\begin{aligned}
& {\left[\begin{array}{lll}
R_{31} & R_{32} & R_{33}
\end{array}\right]^{T}=\left[\begin{array}{lll}
x_{1} & x_{2} & x_{3}
\end{array}\right]^{T}} \\
& \Rightarrow g_{\text {Leg }}\left(q_{i}, v_{\text {joint }}\right)=h_{\text {Leg }}\left(x_{1}, x_{2}, x_{3}\right)
\end{aligned}
$$

\subsubsection{Joint Encoders - Double Support (DS)}

In DS since both feet are on the ground, the trunk along with two legs acts as a closed-loop structure. The joint encoder model for DS is a combination of the joint encoders present on both the legs:

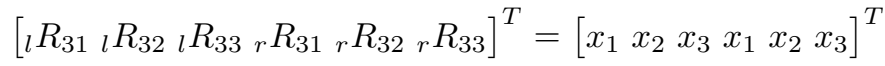

$$
\begin{aligned}
& \Rightarrow g_{\text {BothLegs }}\left({ }_{l} q_{i}, r q_{i}, v_{\text {joint }}\right)=h_{\text {BothLegs }}\left(x_{1}, x_{2}, x_{3}\right)
\end{aligned}
$$

where the left subscripts $l$ and $r$ stand for the left and right leg respectively. But this closed-loop structure leads to kinematic coupling between the joints of both legs. Hence Jacobian $V_{\text {BothLegs }}$ (used to compute the Kalman Gain) in closed loop is given as:

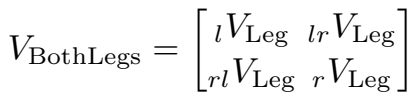

The terms ${ }_{l} V_{\text {Leg }}$ and ${ }_{r} V_{\text {Leg }}$ are calculated from $h_{\text {Leg }}$ as in eq. (9) for the given leg but the terms ${ }_{l r} V_{\text {Leg }}$ and ${ }_{r l} V_{\text {Leg }}$ are calculated using the kinematic model of closed-loop robots. ${ }^{12}$ Inertial frame $b$ is taken as the root of the closed-loop and trunk frame $n$ is attached to the cut joint $k$ (at the hip) such that the two legs act as the two branches of the loop. Applying the kinematic constraint at cut joint $k$ :

$$
[v \omega]^{T}={ }_{l} J_{l} \partial q_{i}={ }_{r}{ }_{r} \partial q_{i}
$$

Using eq. (12), the terms ${ }_{l r} V_{\text {Leg }}$ and ${ }_{r l} V_{\text {Leg }}$ are derived and substituted back in eq. (11) to get $V_{\text {BothLegs }}$ :

$$
\begin{gathered}
{ }_{l r} V_{\text {Leg }}={ }_{l} V_{\text {Leg }} \frac{{ }_{l} \frac{\partial q_{i}}{\partial q_{i}}={ }_{l} V_{\text {Leg } l} J^{-1}{ }_{r} J}{\therefore V_{\text {BothLegs }}}=\left[\begin{array}{cc}
{ }_{l} V_{\text {Leg }} & { }_{l} V_{\text {Leg } l} J^{-1}{ }_{r} J \\
{ }_{r} V_{\operatorname{Leg} r} J^{-1}{ }_{l} J & { }_{r} V_{\text {Leg }}
\end{array}\right]
\end{gathered}
$$




\subsubsection{Generalized Measurement Model of the EKF}

Unlike accelerometer measurements which can be always considered, joint encoder measurements can be used only in certain phases of the humanoid walk. This is because of the fact that the rotation matrix ${ }^{b} R_{0}$ is unknown when the foot is not in complete support (see section 2.2.2). Hence the type of contact a foot has with the ground determines the possibility to calculate the DGM of that leg. The FSRs present at the feet help to determine three types of contact, namely; SS where one foot is in full contact with the ground while the other foot is completely in air; DS where both feet are in full contact with the ground and Intermediate Support (IS) which is any other type of foot contact other than SS and DS. For IS, ${ }^{b} R_{0}$ is unknown but for SS and DS, ${ }^{b} R_{0, \text { Leg }}$ and ${ }^{b} R_{0, \text { BothLegs }}$ are known respectively. Hence depending on the type of contact, different measurements models are constructed by simply combining the individual sensor measurement models that have been derived earlier. It is assumed that there is no slipping or sliding of the legs.

For IS, the general measurement model is simply the accelerometer model as in eq. (7). For SS, the general measurement model (refer eq. (15)) is a combination of accelerometer and joint encoder model of the supporting leg derived using eqs. (7) and (9). For DS, the general measurement model (refer eq. (16)) is a combination of accelerometer and joint encoder model for both legs derived using eqs. (7) and (10).

$$
\begin{aligned}
{\left[g_{\text {Acc }} g_{\text {Leg }}\right]^{T} } & =h_{\mathrm{SS}}\left(x_{1}, x_{2}, x_{3}, v_{\text {noise }}\right) \\
{\left[g_{\text {Acc }} g_{\text {BothLegs }}\right]^{T} } & =h_{\mathrm{DS}}\left(x_{1}, x_{2}, x_{3}, v_{\text {noise }}\right)
\end{aligned}
$$

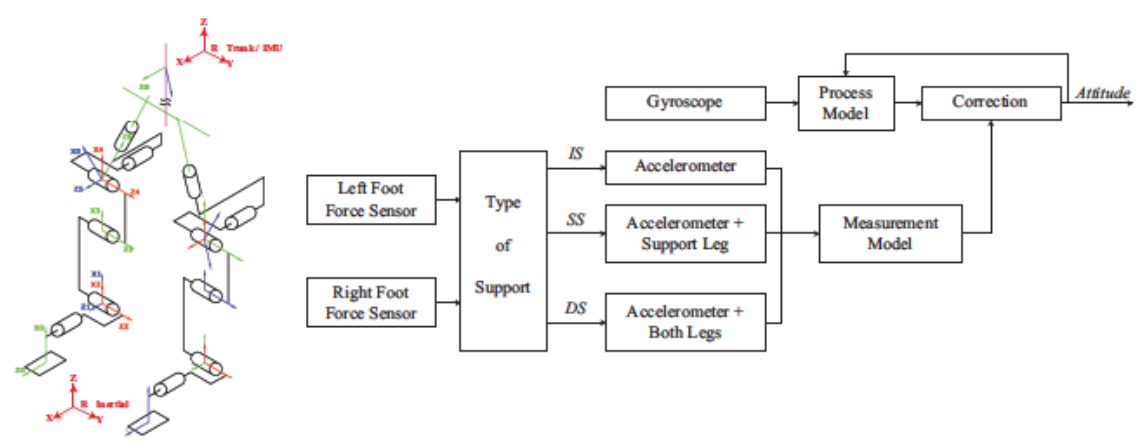

Fig. 1. (a) Modeling the legs and hip of NAO. (b) An overview of the proposed algorithm to estimate the trunk attitude by EKF. 


\section{Results and Discussion}

The developed EKF is applied and tested on the humanoid robot NAO. Three different types of experiments are conducted, namely; 'Bending' where the humanoid bends its trunk with both feet in support by $15 \mathrm{deg}$, stays in this posture and then stands vertically again; 'Walking' where the humanoid walks slowly for a few seconds and then stops and 'Disturbed Walk' where the humanoid is slightly pushed after it starts walking but the push in not strong enough to make it fall. After a few sec. of disturbed walking, it stops and stands still. In all experiments the humanoid starts and stops in a vertical standing position.

\subsection{Comparing Joint Encoder and IMU attitude estimates}

\subsubsection{Joint Encoder data accuracy (Exp - Bending)}

As seen in Fig. 2, the attitude measurement by either the left or right leg joint encoders is quite accurate. In the entire movement since there is no change in roll angle, $\phi$ should be zero. The spikes seen in the roll angle calculated by the joint encoders is due to the sliding of legs. The left leg slides a lot more than the right. However, due to the noise in IMU data the estimated angle $\phi$ diverges.
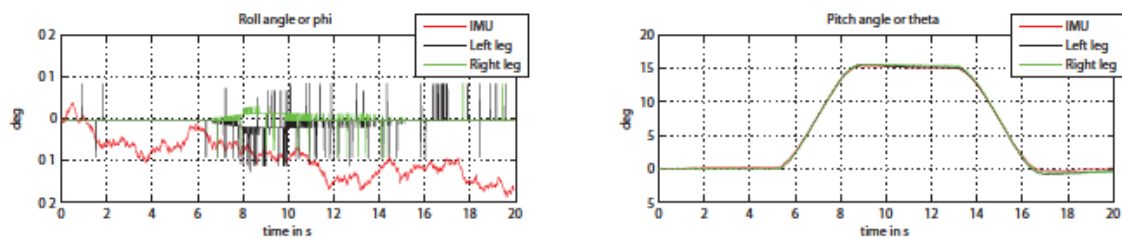

Fig. 2. Trunk attitude estimation by IMU and joint encoders for Bending.

\subsubsection{Detecting type of contact (Exp - Walking)}

It is observed that the attitude estimate given by the IMU and the joint encoders' attitude measure are different when there is a change of contact between the stance leg and the ground. As seen in Fig. 3, the black line (left leg joint encoders' attitude measure) approximately follows the red line (IMU's attitude estimate) when the left leg is in support and stops following the red when left foot goes from stance to swing. At this instant the green line (right leg joint encoders' attitude measure) starts following the red line indicating right leg support. The support phases can be verified by the FSR information from the feet. We believe that the presence of 'rare' 
left foot SS as shown by FSR is either due to the left foot FSR not working properly or too much sliding of the left foot or both reasons.
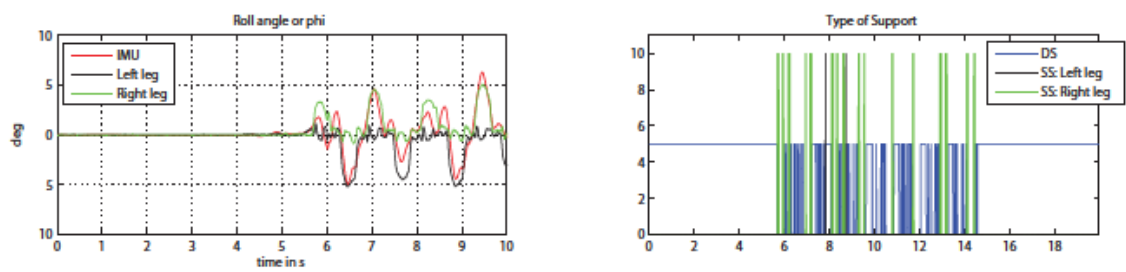

Fig. 3. Change of support by comparing IMU attitude estimate with joint encoders' attitude measure. ('Types of Support' is given by FSRs at feet: $\mathrm{SS}=10$; DS $=5$.)

\subsection{Attitude Estimation by Data Fusion using EKF}

\subsubsection{Walking}

Even during slow walking, the inertial sensors are prone to noise whose effect can be clearly seen in the pitch angle $\theta$ (refer Fig. 4) which overshoots sometimes and does not come back to zero even when motion stops. Data fusion helps to improve the attitude estimate. Another observation seen in the roll angle is the difference for support on right and left leg (clear after $10 \mathrm{sec}$ ) which is probably connected to sliding of one leg which we believe to be the left leg as indicated by the spikes in roll angle in Fig. 2.
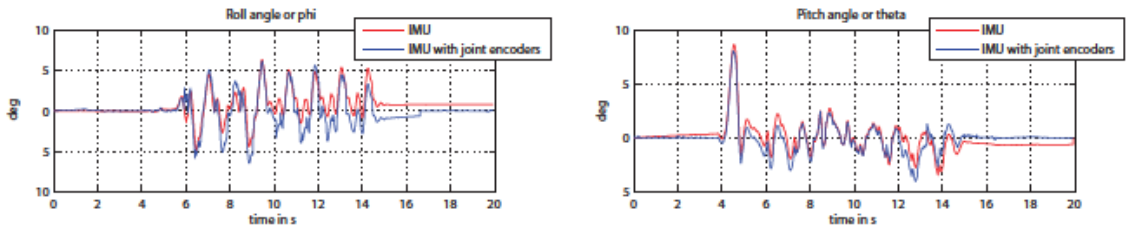

Fig. 4. Attitude estimation of trunk by EKF for Walking.
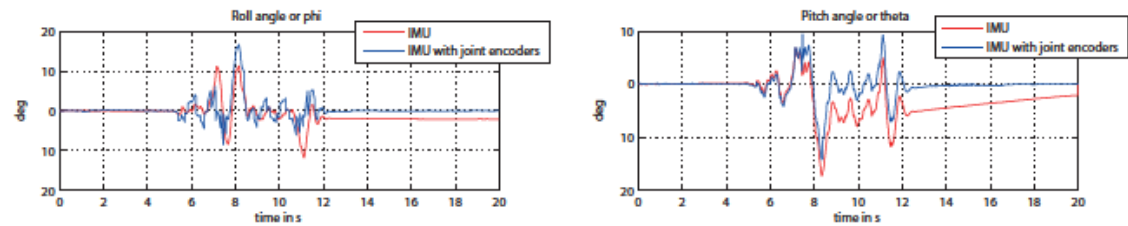

Fig. 5. Attitude estimation of trunk by EKF for Disturbed Walk. 


\subsubsection{Disturbed Walk}

As shown in Fig. 5 the effect of noise that is introduced by the external push (at approx. 7s) is clearly visible in the estimation of roll and pitch angles by using only IMU data. Even after the motion stops, it takes a while for the IMU's attitude estimate to come back to zero. This can lead to a false fall detection. However, data fusion with joint encoders help to correct the effect of inertial noise to give an improved attitude estimate of the humanoid trunk.

\section{Conclusion}

Attiude estimates from inertial sensors that form a part of the IMU cannot be completely trusted as they suffer heavily from various kinds of noise. It is understood that there shall be many external disturbances which the humanoid shall encounter while walking on even or uneven terrains. Poor attitude estimates would risk false fall detections. An effective trunk attitude control capability requires good attitude measures. This can be provided by the joint encoders in the legs which are readily available on a humanoid platform. By fusing the noisy IMU data with joint encoders' data from the legs, an improved trunk attitude estimation can be achieved.

\section{References}

1. O. J. Woodman, An Introduction to Inertial Navigation, Tech. Rep. UCAMCL-TR-696, Comp. Lab., Univ. of Cambridge, (2007).

2. J. R. Solar, J. Moya and I. P. Tsunekawa, Fall detection and management in biped humanoid robots, in ICRA, 2010.

3. L. Lou, X. Xu, J. Cao, Z. Chen and Y. Xu, Sensor fusion-based attitude estimation using low-cost MEMS-IMU for robot navigation, in ITAIC, 2011.

4. C. W. Kang and C. G. Park, Attitude estimation with accelerometers and gyros using fuzzy tuned Kalman filter, in $E C C, 2009$.

5. G. Welch and G. Bishop, An Introduction to the Kalman Filter, Tech. Rep. TR 95-041, Dept. of Comp. Science, Univ. of N. Carolina (2006).

6. F. Caron, E. Duflos, D. Pomorski and P. Vanheeghe, Information Fusion 7, $221(2006)$.

7. J. Crassidis, F. Markley and Y. Cheng, Journal of Guidance, Control and Dynamics 30, 12 (2007).

8. P. S. Maybeck, Stoc. Models, Estimation and Control (Academic Press, 1979).

9. W. Phillips and C. Hailey, Journal Of Aircraft 38, 718 (2001).

10. J. Denavit and R. Hartenberg, Journal of Applied Mechanics 22, 215 (1955).

11. W. Khalil and J. Kleinfinger, A new geometric notation for open and closedloop robots, in ICRA, 1986.

12. W. Khalil and E. Dombre, M., Idn. and Cont. of Robots (H. Penton, 2002). 